Europhys. Lett., 50 (2), pp. 271-277 (2000)

\title{
Unified approach to the tunnel magnetoresistance and the non-equilibrium exchange interaction
}

\author{
C. $\operatorname{Heide}(*)$ and R. J. Elliott \\ University of Oxford, Department of Physics, Theoretical Physics \\ 1 Keble Road, Oxford OX1 3NP, UK
}

(received 25 October 1999; accepted in final form 10 February 2000)

PACS. 75.70.Pa - Giant magnetoresistance.

PACS. 73.40. Rw - Metal-insulator-metal structures.

PACS. 75.10.-b - General theory and models of magnetic ordering.

\begin{abstract}
The tunnel magnetoresistance and its connection to the interlayer exchange interaction is studied in ferromagnet-insulator-ferromagnet junctions. Building on the nonequilibrium Keldysh formalism, we include a contact interaction between localized spins and conduction electrons and derive an expression for the tunnel current that depends on the magnetization of the layers as $\left\langle\boldsymbol{S}_{\mathrm{L}} \cdot \boldsymbol{S}_{\mathrm{R}}\right\rangle$ in correspondence with the exchange coupling. From this expression we also obtain a formula for the tunnel magnetoresistance. At low bias and for systems where one can neglect size-quantization effects Julliere's formula is rederived. The temperature dependence of the dynamical parameters arises from the system of localized spins, its interaction with the conduction electrons, and the thermal reservoirs.
\end{abstract}

There is much evidence to believe that the interaction between the ferromagnetic layers and the effect of giant magnetoresistance (GMR) are directly related. The simplest model which provides a reasonable approximation for transition metals is to assume that the exchange interaction between the magnetic $d$-electrons arises through coupling to the conduction $s$-electrons which then transmit an effective spin-interaction across the spacer through the RudermanKittel-Kasuya-Yosida (RKKY) mechanism. Detailed treatments of this effect, going beyond the simple free-electron picture, had been given in refs. [1-3]. These theories show, as has been found experimentally $[4,5]$, that because of the oscillatory nature of the interaction the coupling between magnetic layers can change in sign with the width of the spacer. On the other hand, the GMR also varies with the width of the spacer and is pronounced in cases when the coupling between the layers is antiferromagnetic, as reviewed in refs. $[6,7]$.

In a recent paper, we pointed out that the tunnel magnetoresistance (TMR) effect and the non-equilibrium exchange interaction (NEXI) are also closely related [8]. We showed that the NEXI in a magnetic tunnel junction (MTJ) is mediated to a large extent by spin-polarized currents. As a function of bias these currents give rise to a change in sign of the coupling. By a comparison with a mean field treatment we found that the spin-polarized currents are USA.

(*) Present address: Physics Department, New York University - 4 Washington Place, New York, NY 10003,

(C) EDP Sciences 
dominated by semi-classical terms and depend to a lesser degree on size-quantization effects. Thus, out of equilibrium the nature of the NEXI may be very different to the typical quantuminterference effect of the equilibrium RKKY-type. It is desirable then to extend our previous treatments at a heuristic level to derive a rigorous mathematical formulation demonstrating the relation between TMR and NEXI in more detail.

For simplicity, we assume a trilayer structure that consists of two planar ferromagnetic layers of thickness $l_{\mathrm{L}(\mathrm{R})}$ and band bottom $V_{\mathrm{L}(\mathrm{R})}$ separated at the interfaces by a dielectric spacer of thickness $d$ and potential $V_{0}$. The conduction electrons are taken to occupy simple spherical conduction bands with energies $\varepsilon_{\mathrm{L}(\mathrm{R})}=\hbar \omega-V_{\mathrm{L}(\mathrm{R})}$. Upon biasing the system, the chemical potentials $\mu_{\mathrm{L}(\mathrm{R})}$ and the band bottoms $V_{\mathrm{L}(\mathrm{R})}$ are both separated by a bias $e V=\mu_{\mathrm{L}}-\mu_{\mathrm{R}}$, while the spacer layer acquires a slope. As the model is translational invariant in the directions perpendicular to the current flow, the analysis can be restricted to one dimension. The results are extended to three dimensions by means of Fourier transforms in $\boldsymbol{k}_{\|}$for the numerical calculations according to refs. [8,9].

Further, we assume that the uncompensated magnetic moments of the $d$-electrons are localized, uniform within the ferromagnets, and the exchange coupling between them and the conduction electrons is isotropic. This leads to an effective interaction of the form

$$
H_{\mathrm{eff}}=-\frac{J}{2} \sum_{p ; \alpha, \beta} \boldsymbol{\sigma}_{\alpha \beta} \cdot \boldsymbol{S}_{p}\left\langle\Psi_{\alpha}^{\dagger}\left(x_{p}\right) \Psi_{\beta}\left(x_{p}\right)\right\rangle
$$

where $J$ is the coupling constant, and $\Psi_{\alpha}^{\dagger}(x)$ and $\Psi_{\alpha}(x)$ are field operators which create and destroy a conduction electron with $\operatorname{spin} \alpha$ at point $x$, respectively; $\boldsymbol{\sigma}_{\alpha \beta}$ is the vector of Pauli matrices, used to represent the spin of the conduction electrons which couple to the local moments $\boldsymbol{S}_{p}$. In order to obtain a proper formulation of the non-equilibrium situation in the system, we employ the Keldysh non-equilibrium perturbation formalism [10] and express the spin-dependent particle density in terms of the Keldysh Green's function $G_{\alpha \beta}^{<}\left(x_{p}, x_{q} ; \omega\right)=$ $\left.i \hbar\left\langle\Psi_{\alpha}^{\dagger}\left(x_{p}\right) \Psi_{\beta}\left(x_{q}\right)\right\rangle\right)$. The details of implementing the non-equilibrium theory to the needs of magnetic layer systems had been discussed in refs. [8,9,11].

It is also necessary to include the effects of diffuse scattering from defects in the ferromagnets. As the dominant contributions to both the current and NEXI are close to the Fermi surface in the quasi-one-dimensional system, we employ the constant relaxation time approximation with $\tau^{-1}=\tau_{\mathrm{i}}^{-1}+\tau_{\mathrm{p}}^{-1}+\tau_{\mathrm{s}}^{-1}$ as in ref. [12]. We note that, although scattering occurs between states with different $\boldsymbol{k}_{\|}$vectors, the exchange coupling between layers of spins is self-averaging [13], and our approximation is tantamount to restoring $\boldsymbol{k}_{\|}$symmetry. The relaxation time $\tau_{\mathrm{i}}$ due to residual impurity scattering is taken to be independent of temperature $T, \tau_{\mathrm{p}}$ due to phonon scattering is inverse proportional to $T$ except at very low temperatures, and $\tau_{\mathrm{s}}$ due to spin disorder scattering via interaction (1) may be approximated as $\tau_{\mathrm{s}}^{-1} \propto J^{2}\left[1-\langle\boldsymbol{S}\rangle^{2} /\left(S^{2}+S\right)\right]$. These different contributions to the bulk scattering are assumed for simplicity to be the same in the left and right ferromagnets and are absorbed in the complex energies $\varepsilon_{\mathrm{L}(\mathrm{R})}^{r}=\varepsilon_{\mathrm{L}(\mathrm{R})}+\Delta+i \hbar \tau^{-1} \equiv\left(\hbar q_{\mathrm{L}(\mathrm{R})}^{r}\right)^{2} / 2 m$. Here, $m$ is the effective mass of the conduction electrons, and $\Delta$ a shift in the band.

The current through the trilayer system depends on its magnetic state which is assumed to be either ferromagnetic or antiferromagnetic. By determining the sign of $E_{\text {ex }}=1 / 2\left(E_{\mathrm{AFM}}-\right.$ $\left.E_{\mathrm{FM}}\right)$, one obtains the stable magnetic configuration. An explicit expression for the NEXI was first derived in ref. [9] and can be written as a sum of the quantum-interference contribution which is finite at equilibrium and a term mediated by the spin-currents, according to ref. [8], 
i.e. $E_{\mathrm{ex}}=\tilde{E}_{\mathrm{ex}}+\bar{E}_{\mathrm{ex}}$, where

$$
\begin{aligned}
& \tilde{E}_{\mathrm{ex}}=-\hbar\left\langle\boldsymbol{S}_{\mathrm{L}} \cdot \boldsymbol{S}_{\mathrm{R}}\right\rangle \int_{-\infty}^{\infty} \frac{\mathrm{d} \omega}{2 \pi}\left(n_{\mathrm{F}}^{\mathrm{L}}+n_{\mathrm{F}}^{\mathrm{R}}\right) \operatorname{Im}\left\{\psi_{\mathrm{LR}}\right\} \\
& \bar{E}_{\mathrm{ex}}=-\hbar\left\langle\boldsymbol{S}_{\mathrm{L}} \cdot \boldsymbol{S}_{\mathrm{R}}\right\rangle \int_{-\infty}^{\infty} \frac{\mathrm{d} \omega}{2 \pi}\left(n_{\mathrm{F}}^{\mathrm{L}}-n_{\mathrm{F}}^{\mathrm{R}}\right) T\left[J_{\mathrm{R}} L_{\mathrm{R}} \operatorname{Re}\left\{\psi_{\mathrm{L}}\right\}-J_{\mathrm{L}} L_{\mathrm{L}} \operatorname{Re}\left\{\psi_{\mathrm{R}}\right\}\right],
\end{aligned}
$$

with $\psi_{\mathrm{L}(\mathrm{R})}=J_{\mathrm{L}(\mathrm{R})} r_{\mathrm{L}(\mathrm{R})}^{r} \zeta_{\mathrm{L}(\mathrm{R})}^{r}$, and $\psi_{\mathrm{LR}}=J_{\mathrm{L}} J_{\mathrm{R}}\left(t^{r}\right)^{2} \zeta_{\mathrm{L}}^{r} \zeta_{\mathrm{R}}^{r}$. We assumed that the interlayer exchange coupling is sufficiently weaker than the direct exchange within the ferromagnets and averaged over their spins $J_{\mathrm{L}(\mathrm{R})}=J \rho_{\mathrm{L}(\mathrm{R})} /\left(\hbar v_{\mathrm{L}(\mathrm{R})}\right)$, where $\rho_{\mathrm{L}(\mathrm{R})}$ is the number of spins per unit volume, and $v_{\mathrm{L}(\mathrm{R})}$ the velocities of the conduction electrons. This also leads to the approximation that $\left\langle\boldsymbol{S}_{\mathrm{L}} \cdot \boldsymbol{S}_{\mathrm{R}}\right\rangle \approx\left\langle S_{\mathrm{L}}^{z}\right\rangle\left\langle S_{\mathrm{R}}^{z}\right\rangle$. The combination of the Fermi functions $n_{\mathrm{F}}^{\mathrm{L}(\mathrm{R})}$ and the form of $\psi_{\mathrm{LR}}$ and $\psi_{\mathrm{L}(\mathrm{R})}$ demonstrate clearly the different nature of $\tilde{E}_{\text {ex }}$ and $\bar{E}_{\text {ex }}$. Apart from the prefactor, the non-local function $\psi_{\mathrm{LR}}$ consists of a transmission amplitude $t^{r}$ through the spacer and an oscillatory part responsible for the quantum-interferences of the RKKY-type, where

$$
\zeta_{\mathrm{L}(\mathrm{R})}^{r}=\frac{1}{2 q_{\mathrm{L}(\mathrm{R})}^{r}}\left(1-e^{2 i q_{\mathrm{L}(\mathrm{R})}^{r} l_{\mathrm{L}(\mathrm{R})}}\right) .
$$

On the other hand, $\psi_{\mathrm{L}(\mathrm{R})}$ is confined to one ferromagnetic layer, where the reflection amplitude $r_{\mathrm{L}(\mathrm{R})}^{r}$ describes the specular reflexion off the interfaces. The influence of the other ferromagnet comes in through $J_{\mathrm{L}(\mathrm{R})} L_{\mathrm{L}(\mathrm{R})}$. $L_{\mathrm{L}(\mathrm{R})}$ diverges for an infinitely large and perfectly conducting ferromagnet, i.e. $l_{\mathrm{L}(\mathrm{R})}$ and $\tau_{\mathrm{L}(\mathrm{R})} \rightarrow \infty$, but in realistic systems we have to take diffuse scattering into account. For $\varepsilon_{\mathrm{L}(\mathrm{R})}^{\mathrm{F}} \gg \hbar \tau^{-1}$ we may write in case of $\hbar \omega \geq V^{\mathrm{L}(\mathrm{R})}$,

$$
L_{\mathrm{L}(\mathrm{R})}=\frac{\lambda_{\mathrm{L}(\mathrm{R})}}{2}\left(1-e^{-2 l_{\mathrm{L}(\mathrm{R})} / \lambda_{\mathrm{L}(\mathrm{R})}}\right),
$$

where $\lambda=v_{\mathrm{L}(\mathrm{R})} \tau$ is the mean free path of the conduction electron spin. Depending on the thickness of the ferromagnets, there are two important limits: the ballistic regime $\lambda_{\mathrm{L}(\mathrm{R})} \gg$ $l_{\mathrm{L}(\mathrm{R})}$, where $L_{\mathrm{L}(\mathrm{R})}=l_{\mathrm{L}(\mathrm{R})}$, and the spin diffusive regime $\lambda_{\mathrm{L}(\mathrm{R})} \ll l_{\mathrm{L}(\mathrm{R})}$, where $L_{\mathrm{L}(\mathrm{R})}=\lambda_{\mathrm{L}(\mathrm{R})} / 2$ and $\zeta_{\mathrm{L}(\mathrm{R})}^{r}=\left(2 q_{\mathrm{L}(\mathrm{R})}^{r}\right)^{-1}$. Since $T=\left|t^{r}\right|$ is the transmission coefficient through the system, $\bar{E}_{\mathrm{ex}}$ is in fact an energy current which may be understood in terms of an effective Zeeman-type energy, where the localised spins interact with an effective field carried by the spin-polarized currents [8].

In case of ferromagnets with a low anisotropy and absence of an external magnetic field, we may use eqs. (2) and (3) to find a closed expression for the current through the trilayer system,

$$
I=I_{0}-\operatorname{sgn}\left(E_{\mathrm{ex}}\right) \Delta I,
$$

where $I_{0}=1 / 2\left(I_{\mathrm{FM}}+I_{\mathrm{AFM}}\right)$ and $\Delta I=1 / 2\left(I_{\mathrm{FM}}-I_{\mathrm{AFM}}\right)$ are defined in analogy to $E_{\mathrm{ex}}$. In this form we can derive $\Delta I$ by a perturbation expansion of the Hamiltonian (1). We calculate it in the same fashion as the spin-current in ref. [8], however with the important difference that the first non-vanishing contribution to the Keldysh Green's function $G_{\alpha \beta}^{<}$is only in second order. After some tedious algebraic manipulations we obtain

$$
\Delta I=-e\left\langle\boldsymbol{S}_{\mathrm{L}} \cdot \boldsymbol{S}_{\mathrm{R}}\right\rangle \int_{-\infty}^{\infty} \frac{\mathrm{d} \omega}{2 \pi}\left(n_{\mathrm{F}}^{\mathrm{L}}-n_{\mathrm{F}}^{\mathrm{R}}\right) T\left[2 \operatorname{Re}\left\{\psi_{\mathrm{L}}\right\} \operatorname{Re}\left\{\psi_{\mathrm{R}}\right\}+\operatorname{Re}\left\{\psi_{\mathrm{LR}}\right\}\right],
$$


$\mathrm{Fe}(100)-\mathrm{Al}_{2} \mathrm{O}_{3}(12)-\mathrm{Fe}(20 . .30)$
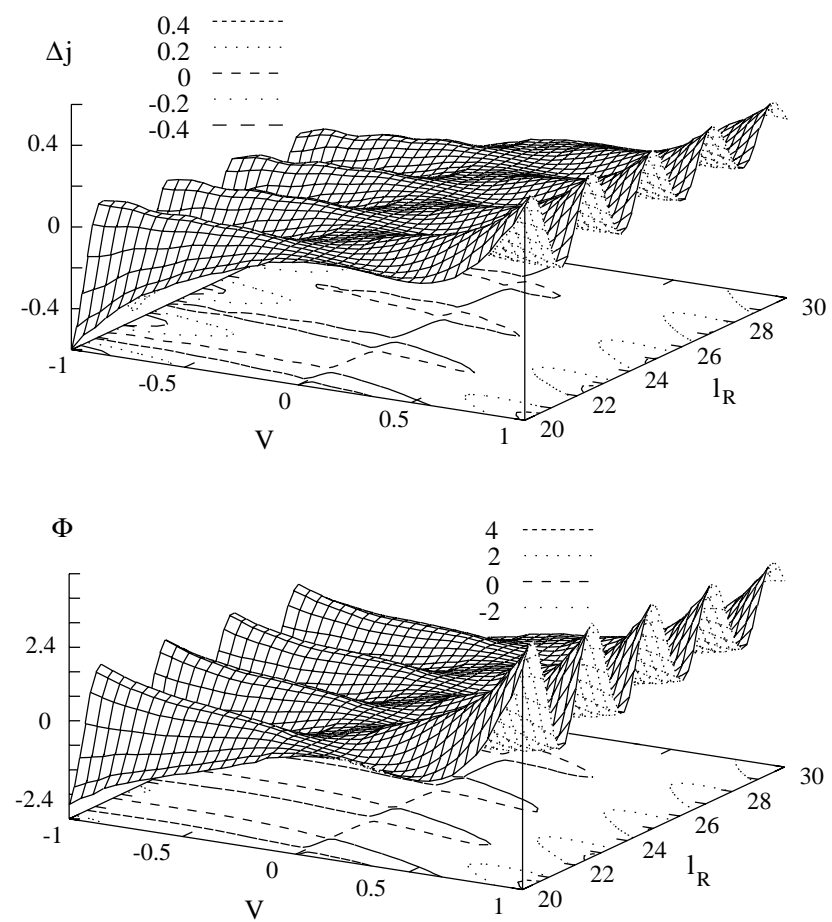

Fig. 1 - Current density $\Delta j\left(\mathrm{~mA} / \mathrm{cm}^{2}\right)$ and NEXI in terms of $\Phi\left(\mathrm{fJ} / \mathrm{cm}^{2}\right)$ as functions of bias and thickness of the right ferromagnet $l_{\mathrm{R}}(\AA)$ in a $\mathrm{Fe}(100 \AA)-\mathrm{Al}_{2} \mathrm{O}_{3}(12 \AA)-\mathrm{Fe}\left(l_{\mathrm{R}}\right)$ junction. The mean free path of the conduction electron spin is taken to be $200 \AA$, and the ratio of barrier height to Fermi energy is $V_{0} / \mu=1.5$. A positive $\Phi$ means ferromagnetic coupling, negative antiferromagnetic.

where $e=|e|$ is the elementary charge of an electron. Comparing $\Delta I$ with $E_{\mathrm{ex}}$, we find that both expressions are closely related. In particular, $\Delta I$ is also proportional to $J^{2}$ and contains the quantum-interference term $\psi_{\mathrm{LR}}$ as well as the individual contributions of the ferromagnets $\psi_{\mathrm{L}(\mathrm{R})}$.

In order to explain the details of the relation between eqs. (2), (3), and (7), we study the behaviour of the current density $\Delta j=\nu^{-1} \Delta I^{3 \mathrm{D}}$ and coupling $\Phi=\nu^{-1} E_{\mathrm{ex}}^{3 \mathrm{D}}$ at zero temperature as functions of the external bias for different MTJ compositions and impurity concentrations, where $\nu$ is the area of the interfaces. For simplicity, we take Fe as the ferromagnetic material on either side of the barrier, and normalize $\left\langle S_{\mathrm{L}(\mathrm{R})}^{z}\right\rangle=S=1$ at $T=0$ to the bulk value. For comparison with possible experiments, e.g. [14], one needs to take into account in addition the thickness dependence for ultra-thin layers [15]. Results for an asymmetric junction are shown in fig. 1. The oscillatory dependence on $l_{\mathrm{R}}$ is notably different for forward and backward bias, but the behaviour of $\Delta j$ and $\Phi$ are remarkably similar. This can be understood by reference to fig. 2, which shows the dependence on the mean free path of the conduction electron spin and compares symmetric and asymmetric junctions. In the symmetric case, $\Delta j$ and $\Phi$ differ considerably and, apart from small terms, are even and odd, respectively, with respect to $\pm V$. To better explain the differences in the behaviour of $\Delta j$ and $\Phi$ for symmetric and asymmetric junctions, we refer back to eqs. (3) and (7). Unlike the current the sign of the NEXI depends 

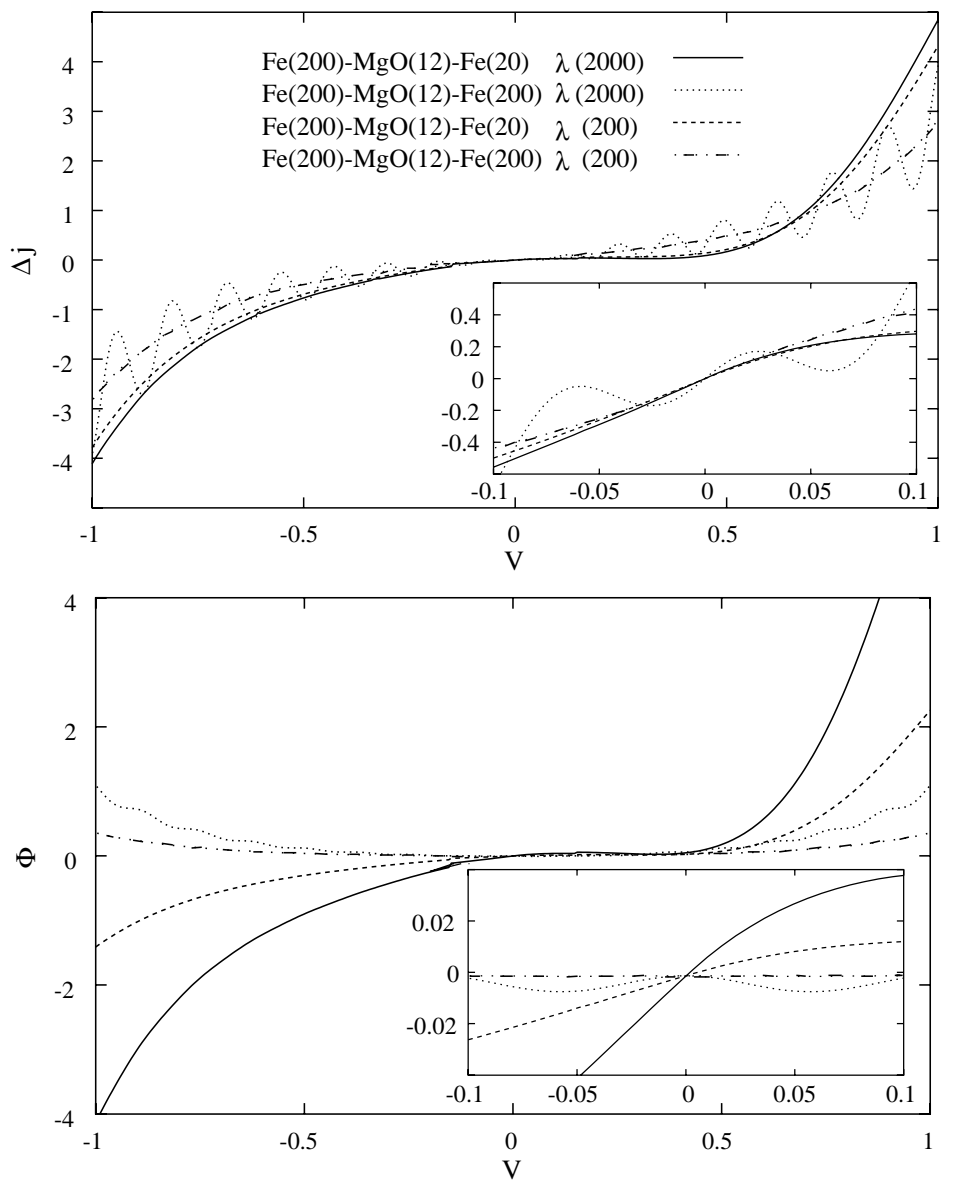

Fig. 2 - Current density $\Delta j\left(\mathrm{~A} / \mathrm{cm}^{2}\right)$ and NEXI in terms of $\Phi\left(\mathrm{pJ} / \mathrm{cm}^{2}\right)$ as functions of bias in a symmetric $\mathrm{Fe}(200 \AA)-\mathrm{MgO}(12 \AA)-\mathrm{Fe}(200 \AA)$ and asymmetric $\mathrm{Fe}(200 \AA)-\mathrm{MgO}(12 \AA)-\mathrm{Fe}(20 \AA)$ junction. The mean free path of the conduction electron spin is taken to be $2000 \AA$ and $200 \AA$, respectively. The ratio of barrier height to Fermi energy $V_{0} / \mu=1.2$.

to a large extent on the relative size of $L_{\mathrm{L}}$ and $L_{\mathrm{R}}$. Only in the symmetric junction these terms nearly cancel, so that in such a case the contribution to the NEXI from eq. (2) are of comparable order (see low bias region of $\Phi$ in fig. 2).

These results show that the effect of a voltage-dependent biasing field is much reduced in the symmetric case, a circumstance which serves to be useful in device design. As in most devices the ferromagnetic materials on either side of the barrier are chosen to be different, leading to an intrinsic asymmetry in the MTJ and thus to a strong voltage-dependent biasing field, it is possible to compensate partly for this effect by just changing the ratio of $l_{\mathrm{L}}$ and $l_{\mathrm{R}}$. On the other hand, the effect of a voltage-dependent biasing field is enhanced for an asymmetric MTJ. It should be in principle possible to switch between the different magnetic configurations by simply reversing the bias in the junction. In the extreme limit of a perfect tunnel barrier, where $\nu=1 \times 1 \mu \mathrm{m}^{2}$, we obtain from the bottom graph of fig. 2 for a bias of $\pm 0.7 \mathrm{~V}$ an exchange coupling of approximately $10^{-20} \mathrm{~J}$. Thus, one could change the orientation of the magnetization in a "soft" layer whose coercive field is smaller than 0.1 Oe and has a 
magnetization of $1 \mathrm{kG}$. Clearly, this limiting case has to be refined considerably for realistic systems as tunnelling is usually limited to a few channels [16] and switching subjected to domain formation [17]. Although for a single channel $\Delta j$ may be significantly larger, $\nu$ is only of the order of $\mathrm{nm}^{2}$. The example, however, shows that the effect of a voltage-dependent biasing field can be quite significant and influence strongly the TMR. In some instances the bias-induced coupling can be ferromagnetic which would make it harder to orient the magnetic electrodes antiparallel. This gives a physical explanation why for certain junctions the TMR decreases significantly with increasing bias $[18,19]$.

From eq. (7) it is also possible to derive an expression for the TMR,

$$
\mathrm{TMR}=\frac{R_{\mathrm{AFM}}-R_{\mathrm{FM}}}{R_{\mathrm{AFM} / \mathrm{FM}}}=\frac{2 \eta}{1 \pm \eta},
$$

where $\eta=\left(\partial_{V} \Delta I\right) /\left(\partial_{V} I_{0}\right)$. As long as transport is ballistic, the TMR depends on quantuminterference effects since $\Delta I$ from eq. (7) enters in eq. (8). We believe that the changes observed recently in the TMR on varying the thickness of one of the ferromagnets may possibly be attributed to such size-quantization effects (compare with $\Delta j$ in fig. 1) [14]. In the spin diffusive regime and under the limiting conditions of a large barrier, i.e. $\psi_{\mathrm{LR}} \approx 0$, we may use the WKB theory to approximate $\Delta I$ in eq. (7), which contains a product of transmission and reflection terms, and $I_{0}$ which, when taken only to the leading order, is equivalent to the usual Landauer formula. If the bias then tends to zero, eq. (8) will lead to TMR $\approx 2 P_{\mathrm{L}} P_{\mathrm{R}}$ as given by Julliere [20]. The values $P_{\mathrm{L}(\mathrm{R})}=\mu_{0} h_{0}^{\mathrm{L}(\mathrm{R})} /\left(2 \varepsilon_{\mathrm{F}}^{\mathrm{L}(\mathrm{R})}\right)$ are the Pauli results for the polarization in one- or quasi-one-dimensional systems, $h_{0}^{\mathrm{L}(\mathrm{R})}=\left\langle S_{\mathrm{L}(\mathrm{R})}\right\rangle J \rho_{\mathrm{L}(\mathrm{R})} /\left(2 \mu_{0}\right)$ is the Weiss field of the ferromagnet, and $\mu_{0}$ the Bohr magneton. For finite bias the WKB result shows a significant decrease of the TMR with increasing bias. However, to obtain a reasonable agreement with the experiments $[18,19,21]$, the effective barrier height of the MTJ ought to be much lower than that obtained from Simmon's theory of tunnelling [18]. This is in correspondence with calculations on the tunnel current that take into account the disorder in the insulator [16]. The details of our analysis shall be presented elsewhere.

Finally, we would like to address in brief the temperature dependence of the NEXI and the TMR. Besides the broadening of the Fermi distributions $n_{\mathrm{F}}^{\mathrm{L}(\mathrm{R})}$, both quantities depend strongly on the thermal fluctuations in the magnetization. For temperatures well below the Curie point of both ferromagnets we may use the spin-wave approximation from the Heisenberg model for the localized moments such that $\left\langle S_{\mathrm{L}(\mathrm{R})}^{z}\right\rangle=S_{\mathrm{L}(\mathrm{R})}-N_{\mathrm{L}(\mathrm{R})}^{-1} \sum_{\boldsymbol{q}} n_{\boldsymbol{q}}^{\mathrm{L}(\mathrm{R})}, n_{\boldsymbol{q}}^{\mathrm{L}(\mathrm{R})}$ are the numbers of excited spin-waves, and $N_{\mathrm{L}(\mathrm{R})}=\nu l_{\mathrm{L}(\mathrm{R})} \rho_{\mathrm{L}(\mathrm{R})}$ the number of spins. Within this approximation the temperature decrease of the NEXI and the TMR has a leading-order term proportional to $T^{3}$ in close agreement with experiments [22]. In addition, this effect is enhanced as spin-dependent scattering increases also as $T^{3}$ which follows from the expression for $\tau_{\mathrm{s}}$. It has to be pointed out, however, that for ferromagnets of less then ten monolayers the Curie temperature decreases almost exponentially as the thickness is reduced [15].

In conclusion, we calculated the difference between the currents in the ferromagnetic and antiferromagnetic states of the system in a perturbative treatment of the $s$ - $d$ interaction. This led to an expression for the tunnel current that depends on the magnetization of the layers as $\left\langle\boldsymbol{S}_{\mathrm{L}} \cdot \boldsymbol{S}_{\mathrm{R}}\right\rangle$ in correspondence with the NEXI. Since the NEXI-induced voltage-dependent biasing fields were shown to affect the current in the MTJ strongly, TMR and NEXI can be regarded as interdependent. It would, therefore, improve the understanding on the nature of the TMR if one could always provide information on the biasing conditions and geometry of the MTJs. In addition, our treatment allowed to deal with the temperature dependence of the MTJ in a straightforward manner. For a more detailed comparison with experiments, we plan 
to include in our approach the important effects of impurity and magnon assisted tunnelling, interface roughness, and proper treatment of the band structure, as has been pointed out by various groups [22-25].

We are indebted to Prof. P. E. Zilberman for numerous discussions. RJE wishes to acknowledge an Emeritus Fellowship from the Leverhulme Foundation and $\mathrm{CH}$ the support provided by the HSK III of the German State and Länder via the DAAD.

\section{REFERENCES}

[1] Bruno P., Phys. Rev. B, 52 (1995) 411.

[2] Stiles M. D., Phys. Rev. B, 48 (1993) 7238.

[3] D’Albuquerque e Castro J., Mathon J., Villeret M. and Edwards D. M., Phys. Rev. $B, 49$ (1994) 16062.

[4] Parkin S. S. P., More N. and Roche K. P., Phys. Rev. Lett., 64 (1990) 2304.

[5] Kawakami R. K. et al., Phys. Rev. Lett., 82 (1999) 4098.

[6] Levy P. M., Giant Magnetoresistance in Magnetic Layered and Granular Materials, Solid State Physics, Vol. 47 (Academic Press, New York) 1994, p. 367.

[7] Gijs M. A. M. and Bauer G. E. W., Adv. Phys., 46 (1997) 285.

[8] Heide C., Elliott R. J. and Wingreen N. S., Phys. Rev. B, 59 (1999) 4287.

[9] Schwabe N. F., Wingreen N. S. and Elliott R. J., Phys. Rev. B, 54 (1996) 12953.

[10] Keldysh L. V., Sov. Phys. JETP, 20 (1965) 1018.

[11] Heide C. and Schwabe N. F., Phys. Rev. B, 57 (1998) 11862.

[12] Elliott R. J. and Wedgwood F. A., Proc. Phys. Soc., 81 (1963) 846.

[13] Levy P. M., Maekawa S. and Bruno P., Phys. Rev. B, 58 (1998) 5588.

[14] Sun J. J. and Freitas P. P., J. Appl. Phys., 85 (1999) 5264.

[15] Erickson R. P. and Mills D. L., Phys. Rev. B, 43 (1991) 11527.

[16] Tsymbal E. Y. and Pettifor D. G., Phys. Rev. B, 58 (1998) 432.

[17] Platt C. L., Dieny B. and Berkowitz A. E., J. Appl. Phys., 81 (1997) 5523.

[18] Moodera J. S., Kinder L. R., Wong T. M. and Meservey R., Phys. Rev. Lett., 74 (1995) 3273.

[19] Montaigne F. et al., Appl. Phys. Lett., 73 (1998) 2829.

[20] Julliere M., Phys. Lett., 54 (1975) 225.

[21] Marley A. C. and Parkin S. S. P., J. Appl. Phys., 81 (1997) 5526.

[22] Shang C. H., Nowak J., Jansen R. and Moodera J. S., Phys. Rev. B, 58 (1998) R2917.

[23] Zhang S., Levy P. M., Marley A. C. and Parkin S. S. P., Phys. Rev. Lett., 79 (1997) 3744.

[24] Tsymbal E. Y. and Pettifor D. G., J. Phys. Condens. Matter, 9 (1997) L411.

[25] Butler W. H. et al., J. Appl. Phys., 81 (1997) 5518. 